\title{
OPERATOR-OPERATOR PADA HIMPUNAN LEMBUT KABUR HESITANT BERNILAI INTERVAL
}

\author{
DILLA FAJRI RASMI, BUKTI GINTING \\ Program Studi S1 Matematika, \\ Fakultas Matematika dan Ilmu Pengetahuan Alam, Universitas Andalas, \\ Kampus UNAND Limau Manis Padang, Indonesia. \\ email : dillafajri648@gmail.com
}

Diterima 9 Maret 2019 Direvisi 7 April 2019 Dipublikasikan 7 Mei 2019

\begin{abstract}
Abstrak. Himpunan lembut kabur hesitant bernilai interval yang diperkenalkan oleh Zhang dkk [12] diterapkan dalam pengambilan keputusan. Menariknya, kita dapat menentukan operasi irisan dan gabungan pada himpunan lembut kabur hesitant bernilai interval dan kita juga dapat menentukan operator-operator baru pada himpunan lembut kabur hesitant bernilai interval, agar kajian tentang himpunan lembut kabur hesitant bernilai interval lebih berkembang. Pada penelitian ini dikaji operasi irisan dan gabungan pada himpunan lembut kabur hesitant bernilai interval dan sifat-sifatnya serta operatoroperator pada himpunan lembut kabur hesitant bernilai interval $\left(\tilde{O}_{1}, \tilde{O}_{2}, \tilde{O}_{3}\right.$ dan $\left.\tilde{O}_{4}\right)$ beserta sifat-sifatnya.

Kata Kunci: Himpunan lembut kabur hesitant bernilai interval, operasi irisan dan gabungan, operator-operator baru
\end{abstract}

\section{Pendahuluan}

Himpunan kabur (fuzzy set/FS) pertama kali di perkenalkan oleh Prof. L.A. Zadeh pada tahun 1965 [12]. Dalam teori himpunan kabur dikaji tentang derajat keanggotaan dari suatu elemen dalam himpunan kabur, yang mana derajat keanggotaan tersebut dinyatakan dengan suatu nilai tunggal dalam interval [0,1]. Pada tahun 2011, Xu dan Xia [10] mendefinisikan konsep dari elemen kabur hesitant, yang dapat dianggap sebagai unit dasar dari himpunan kabur hesitant yang digunakan oleh pembuat keputusan untuk menyatakan keputusan yang ragu-ragu dalam proses pengambilan keputusan.

Pada tahun 2013, Babitha dan Johan [1] mendefinisikan himpunan lembut kabur hesitant (hesitant fuzzy soft set/HFSS). Mereka menerapkan operasi dasar seperti gabungan, irisan, komplemen dan hukum De Morgan ke HFSS. Pada tahun yang sama, Chen dkk [3] memperluas himpunan kabur hesitant ke dalam bentuk himpunan kabur hesitant bernilai interval (IVHFS) dan memperkenalkan konsep dari himpunan kabur hesitant bernilai interval. Pada tahun 2015, Zhang dkk [12] memperkenalkan beberapa operasi seperti komplemen, dan, atau, ring sum, ring product 
pada himpunan lembut kabur hesitant bernilai interval interval valued hesitant fuzzy soft set/(IVHFSS).

Kemudian Thakur dkk [6] mengusulkan empat operator baru $O_{1}, O_{2}, O_{3}, O_{4}$ pada himpunan kabur hesitant. Berdasarkan [6] menarik untuk dikaji tentang operator yang dikenalkan terhadap himpunan kabur hesitant. Pada penelitian ini akan dikaji operasi irisan dan gabungan pada himpunan lembut kabur hesitant bernilai interval dan beberapa sifat-sifatnya. Selanjutnya akan dikaji empat operator $\tilde{O}_{1}$, $\tilde{O}_{2}, \tilde{O}_{3}, \tilde{O}_{4}$ pada himpunan lembut kabur hesitant bernilai interval yang dikaji oleh Borah dan Hazarika [2].

\section{Landasan Teori}

Misalkan U adalah suatu himpunan semesta.

\subsection{Himpunan Lembut Kabur}

Definisi 2.1. [5] Misalkan $P(U)$ adalah suatu himpunan kuasa atas $U, E$ adalah suatu himpunan parameter dan $A \subseteq E$. Himpunan lembut (soft set) $\varepsilon_{A}$ atas $U$ adalah himpunan yang didefinisikan oleh fungsi $\varepsilon_{A}$ yang dapat dinyatakan sebagai himpunan pasangan terurut yang berbentuk:

$$
\varepsilon_{A}=\left\{\left\langle x, \varepsilon_{A}(x)\right\rangle \mid x \in A, \varepsilon_{A}(x) \in P(U)\right\},
$$

dimana $\varepsilon_{A}: A \rightarrow P(U)$ sedemikian sehingga $\varepsilon_{A}=\emptyset$ jika $x \notin A$.

Definisi 2.2. [11] Suatu himpunan kabur A atas U didefinisikan sebagai:

$$
A=\left\{\left\langle x, \mu_{A}(x)\right\rangle \mid x \in U\right\},
$$

dimana $\mu_{A}: U \rightarrow[0,1]$, dan $\mu_{A}(x)$ disebut derajat keanggotaan atas $U$.

Definisi 2.3. [4] Misalkan $I^{u}$ adalah himpunan dari semua himpunan kabur atas $U, E$ adalah suatu himpunan parameter dan $A \subseteq E . \Gamma_{A}$ disebut himpunan lembut kabur jika $\Gamma_{A}: A \rightarrow I^{u}$, yang dapat dinyatakan sebagai himpunan pasangan terurut yang berbentuk:

$$
\Gamma_{A}=\left\{\left\langle x, \Gamma_{A}(x)\right\rangle \mid x \in A, \Gamma_{A}(x) \in I^{u}\right\} .
$$

\subsection{Himpunan Lembut Kabur Hesitant}

Definisi 2.4. [7] Suatu himpunan kabur hesitant $F$ atas $U$ dapat dinyatakan sebagai:

$$
F=\left\{\left\langle x, \mu_{F}(x)\right\rangle \mid x \in U\right\},
$$

dimana $\mu_{F}(x)$ merupakan himpunan dari beberapa nilai-nilai pada interval $[0,1]$. Dalam hal ini dapat ditulis $\mu_{F}(x)=\{\gamma \in[0,1] \mid \gamma$ adalah derajat keanggotaan dari $x \in U\}$. Himpunan $\mu_{F}(x)=\mu$ dinamakan sebagai hesitant fuzzy element (HFE).

Definisi 2.5. [8] Misalkan $E$ adalah suatu himpunan parameter dan $A \subseteq E$, dan $F(U)$ adalah himpunan semua himpunan kabur hesitant atas $U$, maka $F_{A}$ disebut himpunan lembut kabur hesitant atas $U$, dimana $F: A \rightarrow F(U)$. 


\subsection{Himpunan Kabur Hesitant Bernilai Interval}

Definisi 2.6. [3] Misalkan D[0,1] merupakan himpunan dari seluruh subinterval tertutup dari interval $[0,1]$. Suatu himpunan kabur hesitant bernilai interval $\tilde{F}$ atas $U$ dapat dinyatakan sebagai:

$$
\tilde{F}=\left\{\left\langle h, \mu_{\tilde{F}}(h)\right\rangle \mid h \in U\right\},
$$

dimana $\mu_{\tilde{F}}: U \rightarrow D[0,1], \mu_{\tilde{F}}(h)$ disebut derajat keanggotaan dari $h \in U$ pada $\tilde{F}$ dan $\mu_{\tilde{F}}(h)=\tilde{\mu}$ dinamakan sebagai interval-valued hesitant fuzzy element (IVHFE), yang mana $\mu_{\tilde{F}}(h)=\{\tilde{\gamma} \mid \tilde{\gamma} \in D[0,1]\}$. Dalam hal ini $\tilde{\gamma}=\left[\tilde{\gamma}^{L}, \tilde{\gamma}^{U}\right]$ disebut bilangan interval, dimana $\tilde{\gamma}^{L}=\inf \tilde{\gamma}$ dan $\tilde{\gamma}^{U}=\sup \tilde{\gamma}$ berturut-turut menyatakan batas bawah terbesar dan batas atas terkecil dari $\tilde{\gamma}$.

Definisi 2.7. [9] Misalkan $\tilde{a}=\left[\tilde{a}^{L}, \tilde{a}^{U}\right]$ dan $\tilde{b}=\left[\tilde{b}^{L}, \tilde{b}^{U}\right]$ dinamakan dua bilangan interval dan $\lambda \geqslant 0$, maka:

(1) $\tilde{a}=\tilde{b} \Leftrightarrow \tilde{a}^{L}=\tilde{b}^{L}$ dan $\tilde{a}^{U}=\tilde{b}^{U}$;

(2) $\tilde{a}+\tilde{b}=\left[\tilde{a}^{L}+\tilde{b}^{L}, \tilde{a}^{U}+\tilde{b}^{U}\right]$;

(3) $\lambda \tilde{a}=\left[\lambda \tilde{a}^{L}, \lambda \tilde{a}^{U}\right]$, secara khusus $\lambda \tilde{a}^{L}=0$ jika $\lambda=0$.

Definisi 2.8. [9] Misalkan $\tilde{a}=\left[\tilde{a}^{L}, \tilde{a}^{U}\right], \tilde{b}=\left[\tilde{b}^{L}, \tilde{b}^{U}\right]$ dan $l_{a}=\tilde{a}^{U}-\tilde{a}^{L}, l_{b}=\tilde{b}^{U}-\tilde{b}^{L}$, maka nilai kemungkinan (possibility) dari $\tilde{a} \geqslant \tilde{b}$ didefinisikan sebagai:

$$
p(\tilde{a} \geqslant \tilde{b})=\max \left\{1-\max \left\{\frac{\tilde{b}^{U}-\tilde{a}^{L}}{l_{\tilde{a}}+l_{\tilde{b}}}, 0\right\}, 0\right\} .
$$

Definisi di atas digunakan untuk membandingkan dua bilangan interval dan untuk menentukan tingkatannya.

Definisi 2.9. [3] Misalkan $\tilde{\mu}, \tilde{\mu}_{1}$ dan $\tilde{\mu}_{2}$ merupakan tiga IVHFE, maka:

(1) $\tilde{\mu}^{c}=\left\{\left[1-\tilde{\gamma}^{U}, 1-\tilde{\gamma}^{L}\right] \mid \tilde{\gamma} \in \tilde{\mu}\right\}$;

(2) $\tilde{\mu}_{1} \cup \tilde{\mu}_{2}=\left\{\left[\max \left\{\tilde{\gamma}_{1}^{L}, \tilde{\gamma}_{2}^{L}\right\}, \max \left\{\tilde{\gamma}_{1}^{U}, \tilde{\gamma}_{2}^{U}\right\}\right] \mid \tilde{\gamma}_{1} \in \tilde{\mu}_{1}, \tilde{\gamma}_{2} \in \tilde{\mu}_{2}\right\}$;

(3) $\tilde{\mu}_{1} \cap \tilde{\mu}_{2}=\left\{\left[\min \left\{\tilde{\gamma}_{1}^{L}, \tilde{\gamma}_{2}^{L}\right\}, \min \left\{\tilde{\gamma}_{1}^{U}, \tilde{\gamma}_{2}^{U}\right\}\right] \mid \tilde{\gamma}_{1} \in \tilde{\mu}_{1}, \tilde{\gamma}_{2} \in \tilde{\mu}_{2}\right\}$;

(4) $\tilde{\mu}_{1} \oplus \tilde{\mu}_{2}=\left\{\left[\tilde{\gamma}_{1}^{L}+\tilde{\gamma}_{2}^{L}-\tilde{\gamma}_{1}^{L} \cdot \tilde{\gamma}_{2}^{L}, \tilde{\gamma}_{1}^{U}+\tilde{\gamma}_{2}^{U}-\tilde{\gamma}_{1}^{U} \cdot \tilde{\gamma}_{2}^{U}\right] \mid \tilde{\gamma}_{1} \in \tilde{\mu}_{1}, \tilde{\gamma}_{2} \in \tilde{\mu}_{2}\right\}$;

(5) $\tilde{\mu}_{1} \otimes \tilde{\mu}_{2}=\left\{\left[\tilde{\gamma}_{1}^{L} \cdot \tilde{\gamma}_{2}^{L}, \tilde{\gamma}_{1}^{U} \cdot \tilde{\gamma}_{2}^{U}\right] \mid \tilde{\gamma}_{1} \in \tilde{\mu}_{1}, \tilde{\gamma}_{2} \in \tilde{\mu}_{2}\right\}$.

\subsection{Himpunan Lembut Kabur Hesitant Bernilai Interval}

Definisi 2.10. [12] Misalkan E adalah suatu himpunan parameter dan $A \subseteq$ E. Jika $\tilde{F}$ adalah suatu pemetaan yang diberikan oleh $\tilde{F}: A \rightarrow I V H F(U)$, dimana $\operatorname{IVHF}(U)$ adalah himpunan dari semua himpunan kabur hesitant bernilai interval atas $U$. Himpunan $\tilde{F}_{A}$ dikatakan himpunan lembut kabur hesitant bernilai interval atas $U$ dapat ditulis sebagai:

$$
\tilde{F}_{A}=\{\langle e, \tilde{F}(e)\rangle|e \in A, \tilde{F}(e) \in I V H F(U)\rangle\} .
$$

Suatu himpunan lembut kabur hesitant bernilai interval $\tilde{F}_{A}$ merupakan suatu pemetaan dari himpunan parameter A ke IVHF $(U)$. Misalkan e $\in$ A, maka $\tilde{F}(e)$ adalah himpunan kabur hesitant bernilai interval dan $\tilde{F}(e)$ dapat ditulis sebagai:

$$
\tilde{F}(e)=\left\{\left\langle h, \mu_{\tilde{F}(e)}(h)\right\rangle \mid h \in U\right\} .
$$


$\mu_{\tilde{F}(e)}(h)$ adalah kumpulan beberapa subinterval tertutup dari interval $[0,1]$.

Definisi 2.11. [12] Komplemen dari himpunan lembut kabur hesitant bernilai interval $\tilde{F}_{A}$ dinotasikan dengan $\tilde{F}_{A}^{c}$, dimana $\tilde{F}^{c}(e)=(\tilde{F}(e))^{c}, \forall e \in A$, sehingga $\tilde{F}^{c}(e)=\left\{\left\langle h, \mu_{\tilde{F}^{c}(e)}(h)\right\rangle \mid h \in U\right\}$.

Definisi 2.12. [12] Suatu himpunan lembut kabur hesitant bernilai interval dikatakan himpunan lembut kabur hesitant bernilai interval kosong, dinotasikan dengan $\tilde{\phi_{A}}$, dimana $\tilde{\phi}(e)=\left\{\left\langle h, \mu_{\tilde{\phi}(e)}(h)\right\rangle \mid h \in U\right\}=\{\langle h,\{[0,0]\}\rangle \mid h \in U\}, \forall e \in A$.

Definisi 2.13. [12] Suatu himpunan lembut kabur hesitant bernilai interval dikatakan himpunan lemut kabur hesitant bernilai interval penuh, dinotasikan dengan $\tilde{E_{A}}$, dimana $\tilde{E}(e)=\left\{\left\langle h, \mu_{\tilde{E}(e)}(h)\right\rangle \mid h \in U\right\}=\{\langle h,\{[1,1]\}\rangle \mid h \in U\}, \forall e \in A$.

Definisi 2.14. [12] Operasi ring sum pada dua himpunan lembut kabur hesitant bernilai interval $\tilde{F}_{A}$ dan $\tilde{G}_{A}$ atas $(U, E)$, dinotasikan dengan $\tilde{F}_{A} \oplus \tilde{G}_{A}=\tilde{H}_{A}$, dimana

$$
\tilde{H}(e)=\left\{\left\langle h, \mu_{\tilde{H}(e)}(h)\right\rangle \mid h \in U\right\}=\left\{\left\langle h, \mu_{\tilde{F}(e)}(h) \oplus \mu_{\tilde{G}(e)}(h)\right\rangle \mid h \in U\right\}, \forall e \in A .
$$

Definisi 2.15. [12] Operasi ring product pada dua himpunan lembut kabur hesitant bernilai interval $\tilde{F_{A}}$ dan $\tilde{G_{A}}$ atas $(U, E)$, dinotasikan dengan $\tilde{F_{A}} \otimes \tilde{G_{A}}=\tilde{H}_{A}$, dimana

$$
\tilde{H}(e)=\left\{\left\langle h, \mu_{\tilde{H}(e)}(h)\right\rangle \mid h \in U\right\}=\left\{\left\langle h, \mu_{\tilde{F}(e)}(h) \otimes \mu_{\tilde{G}(e)}(h)\right\rangle \mid h \in U\right\}, \forall e \in A .
$$

\section{Pembahasan}

Pada bagian ini akan dijelaskan tentang operasi irisan dan gabungan pada himpunan lembut kabur hesitant bernilai interval beserta sifat-sifatnya dan operator-operator pada himpunan lembut kabur hesitant bernilai interval beserta sifat-sifatnya.

\subsection{Operasi Irisan dan Gabungan pada Himpunan Lembut Kabur Hesitant Bernilai Interval}

Borah dan Hazarika [2] mengkaji operasi irisan dan gabungan pada himpunan lembut kabur hesitant bernilai interval beserta sifat-sifatnya.

Definisi 3.1. [2] Gabungan dari dua himpunan lembut kabur hesitant bernilai interval $\tilde{F}_{A}$ dan $\tilde{G_{B}}$ atas $(U, E)$ adalah himpunan lembut kabur hesitant bernilai interval $\tilde{H}_{C}$, dimana $C=A \cup B$ dan $\forall e \in C$,

$$
\mu_{\tilde{H}(e)}(h)=\left\{\begin{array}{cc}
\mu_{\tilde{F}(e)}(h), & e \in A-B, h \in U, \\
\mu_{\tilde{G}(e)}(h), & e \in B-A, h \in U, \\
\mu_{\tilde{F}(e)}(h) \cup \mu_{\tilde{G}(e)}(h), & e \in A \cap B, h \in U .
\end{array}\right.
$$

Gabungan dari $\tilde{F_{A}}$ dan $\tilde{G_{B}}$ dinotasikan dengan $\tilde{F_{A}} \tilde{\cup} \tilde{G_{B}}=\tilde{H}_{C}$.

$$
\tilde{H}_{C}=\{\langle e, \tilde{H}(e)\rangle|e \in A, \tilde{H}(e) \in I V H F(U)\rangle\} \text { dengan } \tilde{H}(e)=\left\{\left\langle h, \mu_{\tilde{H}(e)}(h)\right\rangle \mid h \in U\right\} .
$$

Definisi 3.2. [2] Irisan dari dua himpunan lembut kabur hesitant bernilai interval $\tilde{F_{A}}$ dan $\tilde{G_{B}}$ dengan $A \cap B \neq \emptyset$ atas $(U, E)$ adalah himpunan lembut kabur hesitant bernilai interval $\tilde{H}_{C}$, dimana $C=A \cap B$ dan $\forall e \in C, \mu_{\tilde{H}(e)}(h)=\mu_{\tilde{F}(e)}(h) \cap$ 
$\mu_{\tilde{G}(e)}(h)$. Irisan dari $\tilde{F_{A}}$ dan $\tilde{G_{B}}$ dinotasikan dengan $\tilde{F_{A}} \tilde{\cap} \tilde{G_{B}}=\tilde{H_{C}} \cdot \tilde{H}_{C}=$ $\{\langle e, \tilde{H}(e)\rangle|e \in A, \tilde{H}(e) \in I V H F(U)\rangle\}$ dimana $\tilde{H}(e)=\left\{\left\langle h, \mu_{\tilde{H}(e)}(h)\right\rangle \mid h \in U\right\}$.

Proposisi 3.3. [2] Misalkan $\tilde{F_{A}}$ merupakan himpunan lembut kabur hesitant bernilai interval, maka berikut ini berlaku:

(1) $\tilde{F_{A}} \tilde{\cup} \tilde{F}_{A}=\tilde{F}_{A}$,

(2) $\tilde{F_{A}} \tilde{\cap} \tilde{F}_{A}=\tilde{F}_{A}$,

(3) $\tilde{F}_{A} \tilde{\cup} \tilde{\phi}_{A}=\tilde{F}_{A}$,

(4) $\tilde{F}_{A} \tilde{\cap} \tilde{\phi}_{A}=\tilde{\phi}_{A}$,

(5) $\tilde{F}_{A} \tilde{\cup} \tilde{E}_{A}=\tilde{E}_{A}$,

(6) $\tilde{F_{A}} \tilde{\cap} \tilde{E}_{A}=\tilde{F}_{A}$.

Bukti. Akan dibuktikan $\tilde{F_{A}} \tilde{\cup} \tilde{F}_{A}=\tilde{F_{A}}$. Untuk kasus lain, pembuktian dilakukan dengan cara yang serupa.

Misalkan $\tilde{F}_{A}=\{\langle e, \tilde{F}(e)\rangle \mid e \in A, \tilde{F}(e) \in I V H F(U)\}$, dimana $\tilde{F}(e)=$ $\left\{\left\langle h, \mu_{\tilde{F}(e)}(h)\right\rangle \mid h \in U\right\}$. Berdasarkan Definisi 3.1, misalkan $\tilde{F}_{A} \tilde{\cup} \tilde{F}_{A}=\tilde{H}_{C}$, dimana $C=A \cup A=A$ dan $\forall e \in C$ diperoleh

$$
\begin{aligned}
\mu_{\tilde{H}(e)}(h) & =\mu_{\tilde{F}(e)}(h) \cup \mu_{\tilde{F}(e)}(h) \\
& =\mu_{\tilde{F}(e)}(h) .
\end{aligned}
$$

Oleh karena $\mu_{\tilde{H}(e)}(h)=\mu_{\tilde{F}(e)}(h), \forall e \in A$, maka terbukti bahwa $\tilde{F}_{A} \tilde{\cup} \tilde{F}_{A}=\tilde{F}_{A}$.

Proposisi 3.4. [2] Misalkan $\tilde{F_{A}}$ dan $\tilde{G_{A}}$ merupakan dua himpunan lembut kabur hesitant bernilai interval, maka berlaku hukum de Morgan sebagai berikut:

(1) $\left(\tilde{F}_{A} \tilde{\cup} \tilde{G}_{A}\right)^{c}=\tilde{F}_{A}^{c} \tilde{\cap} \tilde{G}_{A}^{c}$,

(2) $\left(\tilde{F}_{A} \tilde{\cap} \tilde{G}_{A}\right)^{c}=\tilde{F}_{A}^{c} \tilde{\cup} \tilde{G}_{A}^{c}$.

Bukti. Misalkan $\tilde{F}_{A}=\{\langle e, \tilde{F}(e)\rangle \mid e \in A, \tilde{F}(e) \in I V H F(U)\}$, dimana $\tilde{F}(e)=$ $\left\{\left\langle h, \mu_{\tilde{F}(e)}(h)\right\rangle \mid h \in U\right\}$ dan $\tilde{G_{A}}=\{\langle e, \tilde{G}(e)\rangle \mid e \in A, \tilde{G}(e) \in I V H F(U)\}$, dimana $\tilde{G}(e)=\left\{\left\langle h, \mu_{\tilde{G}(e)}(h)\right\rangle \mid h \in U\right\}$.

Berdasarkan Definisi 2.11, $\tilde{F}_{A}^{c}=\left\{\left\langle e, \tilde{F}^{c}(e)\right\rangle \mid e \in A, \tilde{F}^{c}(e) \in I V H F(U)\right\}$, dimana $\tilde{F}^{c}(e)=\left\{\left\langle h, \mu_{\tilde{F}^{c}(e)}(h)\right\rangle \mid h \in U\right\}$ dan $\tilde{G}_{A}^{c}=\left\{\left\langle e, \tilde{G}^{c}(e)\right\rangle \mid e \in A, \tilde{G}^{c}(e) \in I V H F(U)\right\}$, dimana $\tilde{G}^{c}(e)=\left\{\left\langle h, \mu_{\tilde{G}^{c}(e)}(h)\right\rangle \mid h \in U\right\}$.

(1) Akan dibuktikan $\left(\tilde{F}_{A} \tilde{\cup} \tilde{G}_{A}\right)^{c}=\tilde{F}_{A}^{c} \tilde{\cap} \tilde{G}_{A}^{c}$.

(i) Berdasarkan Definisi 3.1, misalkan $\tilde{F_{A}} \tilde{\cup} \tilde{G_{A}}=\tilde{H}_{C}$, dimana $C=A \cup A=A$ dan $\forall e \in C$ diperoleh $\mu_{\tilde{H}(e)}(h)=\mu_{\tilde{F}(e)}(h) \tilde{\cup} \mu_{\tilde{G}(e)}(h)$.

Misalkan $\left(\tilde{F_{A}} \tilde{\cup} \tilde{G}_{A}\right)^{c}=\tilde{H}_{C}^{c}$, dimana $C=A \cup A=A$ dan $\forall e \in C$ diperoleh $\mu_{\tilde{H}^{c}(e)}(h)=\left(\mu_{\tilde{F}(e)}(h) \cup \mu_{\tilde{G}(e)}(h)\right)^{c}$. 
(ii) Berdasarkan Definisi 3.2, misalkan $\tilde{F}_{A}^{c} \tilde{\cap} \tilde{G}_{A}^{c}=\tilde{I_{D}}$, dimana $D=A \cap A=A$ dan $\forall e \in D$ diperoleh

$$
\begin{aligned}
\mu_{\tilde{I}(e)}(h) & =\mu_{\tilde{F}^{c}(e)}(h) \cap \mu_{\tilde{G}^{c}(e)}(h) \\
& =\left(\mu_{\tilde{F}(e)}(h) \cup \mu_{\tilde{G}(e)}(h)\right)^{c} \\
& =\mu_{\tilde{H}^{c}(e)}(h) .
\end{aligned}
$$

Oleh karena $\mu_{\tilde{I}(e)}(h)=\mu_{\tilde{H}^{c}(e)}(h), \forall e \in A, h \in U$ maka terbukti bahwa $\left(\tilde{F_{A}} \tilde{\cup} \tilde{G}_{A}\right)^{c}=\tilde{F}_{A}^{c} \tilde{\cap} \tilde{G}_{A}^{c}$.

Proposisi 3.5. [2] Misalkan $\tilde{F_{A}}, \tilde{G_{B}}$ dan $\tilde{H}_{C}$ merupakan tiga himpunan lembut kabur hesitant bernilai interval, maka berikut ini berlaku:

(1) $\tilde{F_{A}} \tilde{\cup} \tilde{G_{B}}=\tilde{G_{B}} \tilde{\cup} \tilde{F_{A}}$,

(2) $\tilde{F_{A}} \tilde{\cap} \tilde{G_{B}}=\tilde{G_{B}} \tilde{\cap} \tilde{F_{A}}$,

(3) $\tilde{F}_{A} \tilde{\cup}\left(\tilde{G_{B}} \tilde{\cup} \tilde{H}_{C}\right)=\left(\tilde{F}_{A} \tilde{\cup} \tilde{G}_{B}\right) \tilde{\cup} \tilde{H}_{C}$,

(4) $\tilde{F}_{A} \tilde{\cap}\left(\tilde{G_{B}} \tilde{\cap} \tilde{H}_{C}\right)=\left(\tilde{F}_{A} \tilde{\cap} \tilde{G}_{B}\right) \tilde{\cap} \tilde{H}_{C}$.

Bukti. Misalkan $\tilde{F}_{A}=\{\langle e, \tilde{F}(e)\rangle \mid e \in A, \tilde{F}(e) \in I V H F(U)\}$, dimana $\tilde{F}(e)=$ $\left\{\left\langle h, \mu_{\tilde{F}(e)}(h)\right\rangle \mid h \in U\right\}$ dan $\tilde{G}_{A}=\{\langle e, \tilde{G}(e)\rangle \mid e \in A, \tilde{G}(e) \in I V H F(U)\}$, dimana $\tilde{G}(e)=\left\{\left\langle h, \mu_{\tilde{G}(e)}(h)\right\rangle \mid h \in U\right\}$.

(1) Akan dibuktikan $\tilde{F_{A}} \tilde{\cup} \tilde{G_{B}}=\tilde{G_{B}} \tilde{\cup} \tilde{F_{A}}$.

(i) Berdasarkan Definisi 3.1, misalkan $\tilde{F_{A}} \tilde{\cup} \tilde{G}_{B}=\tilde{H}_{C}$, dimana $C=A \cup B$ dan $\forall e \in C$ diperoleh

$$
\mu_{\tilde{H}(e)}(h)= \begin{cases}\mu_{\tilde{F}(e)}(h), & \text { jika } e \in A-B, h \in U . \\ \mu_{\tilde{G}(e)}(h), & \text { jika } e \in B-A, h \in U . \\ \mu_{\tilde{F}(e)}(h) \cup \mu_{\tilde{G}(e)}(h), & \text { jika } e \in A \cap B, h \in U .\end{cases}
$$

(ii) Berdasarkan Definisi 3.1, misalkan $\tilde{G_{B}} \tilde{\cup} \tilde{F_{A}}=\tilde{I_{D}}$, dimana $D=B \cup A=$ $A \cup B=C$ dan $\forall e \in C$ diperoleh

$$
\mu_{\tilde{I}(e)}(h)= \begin{cases}\mu_{\tilde{F}(e)}(h), & \text { jika } e \in A-B, h \in U . \\ \mu_{\tilde{G}(e)}(h), & \text { jika } e \in B-A, h \in U . \\ \mu_{\tilde{G}(e)}(h) \cup \mu_{\tilde{F}(e)}(h)=\mu_{\tilde{F}(e)}(h) \cup \mu_{\tilde{G}(e)}(h), & \text { jika } e \in A \cap B, h \in U .\end{cases}
$$

Oleh karena $\mu_{\tilde{H}(e)}(h)=\mu_{\tilde{I}(e)}(h), \forall e \in C$ maka terbukti bahwa $\tilde{F_{A}} \tilde{\cup} \tilde{G_{B}}=$ $\tilde{G_{B}} \tilde{\cup} \tilde{F}_{A}$.

Proposisi 3.6. [2] Misalkan $\tilde{F_{A}}, \tilde{G_{B}}$ dan $\tilde{H}_{C}$ merupakan tiga himpunan lembut kabur hesitant bernilai interval, maka berikut ini berlaku:

(1) $\tilde{F}_{A} \tilde{\cup}\left(\tilde{G_{B}} \tilde{\cap} \tilde{H}_{C}\right)=\left(\tilde{F}_{A} \tilde{\cup} \tilde{G_{B}}\right) \tilde{\cap}\left(\tilde{F}_{A} \tilde{\cup} \tilde{H}_{C}\right)$,

(2) $\tilde{F_{A}} \tilde{\cap}\left(\tilde{G_{B}} \tilde{\cup} \tilde{H}_{C}\right)=\left(\tilde{F_{A}} \tilde{\cap} \tilde{G_{B}}\right) \tilde{\cup}\left(\tilde{F_{A}} \tilde{\cap} \tilde{H}_{C}\right)$. 


\subsection{Operator-operator Baru pada Anggota Himpunan Lembut Kabur Hesitant Bernilai Interval}

Pada tahun 2014, Takur dkk [6] telah mengusulkan terlebih dahulu empat operator baru $\mathrm{O}_{1}, \mathrm{O}_{2}, \mathrm{O}_{3}, \mathrm{O}_{4}$ pada himpunan kabur hesitant. Kemudian pada tahun 2018, Borah [2] memperkenalkan empat operator baru $\tilde{O}_{1}, \tilde{O}_{2}, \tilde{O}_{3}, \tilde{O}_{4}$ pada himpunan lembut kabur hesitant bernilai interval. Pada bagian ini akan diuraikan operatoroperator baru yaitu $\tilde{O}_{1}, \tilde{O}_{2}, \tilde{O}_{3}, \tilde{O}_{4}$ pada himpunan lembut kabur hesitant bernilai interval.

Definisi 3.7. [2] Misalkan $\tilde{\mu}_{1}, \tilde{\mu}_{2}$ merupakan anggota himpunan lembut kabur hesitant bernilai interval dari himpunan parameter yang sama, maka:
(i) $\tilde{\mu}_{1} \tilde{O}_{1} \tilde{\mu}_{2}=\bigcup_{\tilde{\gamma}_{1} \in \tilde{\mu}_{1}, \tilde{\gamma}_{2} \in \tilde{\mu}_{2}}\left[\frac{\left|\tilde{\gamma}_{1}^{L}-\tilde{\gamma}_{2}^{L}\right|}{1+\left|\tilde{\gamma}_{1}^{L}-\tilde{\gamma}_{2}^{L}\right|}, \frac{\left|\tilde{\gamma}_{1}^{U}-\tilde{\gamma}_{2}^{U}\right|}{1+\left|\tilde{\gamma}_{1}^{U}-\tilde{\gamma}_{2}^{U}\right|}\right]$,
(ii) $\tilde{\mu}_{1} \tilde{O}_{2} \tilde{\mu}_{2}=\bigcup_{\tilde{\gamma}_{1} \in \tilde{\mu}_{1}, \tilde{\gamma}_{2} \in \tilde{\mu}_{2}}\left[\frac{\left|\tilde{\gamma}_{1}^{L}-\tilde{\gamma}_{2}^{L}\right|}{1+2\left|\tilde{\gamma}_{1}^{L}-\tilde{\gamma}_{2}^{L}\right|}, \frac{\left|\tilde{\gamma}_{1}^{U}-\tilde{\gamma}_{2}^{U}\right|}{1+2\left|\tilde{\gamma}_{1}^{U}-\tilde{\gamma}_{2}^{U}\right|}\right]$,
(iii) $\tilde{\mu}_{1} \tilde{O}_{3} \tilde{\mu}_{2}=\bigcup_{\tilde{\gamma}_{1} \in \tilde{\mu}_{1}, \tilde{\gamma}_{2} \in \tilde{\mu}_{2}}\left[\frac{\left|\tilde{\gamma}_{1}^{L}-\tilde{\gamma}_{2}^{L}\right|}{2}, \frac{\left|\tilde{\gamma}_{1}^{U}-\tilde{\gamma}_{2}^{U}\right|}{2}\right]$,
(iv) $\tilde{\mu}_{1} \tilde{O}_{4} \tilde{\mu}_{2}=\bigcup_{\tilde{\gamma}_{1} \in \tilde{\mu}_{1}, \tilde{\gamma}_{2} \in \tilde{\mu}_{2}}\left[\frac{\left|\tilde{\gamma}_{1}^{L} \cdot \tilde{\gamma}_{2}^{L}\right|}{2}, \frac{\left|\tilde{\gamma}_{1}^{U} \cdot \tilde{\gamma}_{2}^{U}\right|}{2}\right]$.

Proposisi 3.8. [2] Misalkan $\tilde{\mu}_{1}$ dan $\tilde{\mu}_{2}$ merupakan anggota himpunan lembut kabur hesitant bernilai interval, maka berikut ini berlaku:

(i) $\left(\tilde{\mu}_{1} \oplus \tilde{\mu}_{2}\right) \cap\left(\tilde{\mu}_{1} \tilde{O}_{1} \tilde{\mu}_{2}\right)=\tilde{\mu}_{1} \tilde{O}_{1} \tilde{\mu}_{2}$,

(ii) $\left(\tilde{\mu}_{1} \oplus \tilde{\mu}_{2}\right) \cup\left(\tilde{\mu}_{1} \tilde{O}_{1} \tilde{\mu}_{2}\right)=\tilde{\mu}_{1} \oplus \tilde{\mu}_{2}$,

(iii) $\left(\tilde{\mu}_{1} \otimes \tilde{\mu}_{2}\right) \cap\left(\tilde{\mu}_{1} \tilde{O}_{1} \tilde{\mu}_{2}\right)=\tilde{\mu}_{1} \tilde{O}_{1} \tilde{\mu}_{2}$,

(iv) $\left(\tilde{\mu}_{1} \otimes \tilde{\mu}_{2}\right) \cup\left(\tilde{\mu}_{1} \tilde{O}_{1} \tilde{\mu}_{2}\right)=\tilde{\mu}_{1} \otimes \tilde{\mu}_{2}$.

\section{Bukti.}

(i) Akan dibuktikan $\left(\tilde{\mu}_{1} \oplus \tilde{\mu}_{2}\right) \cap\left(\tilde{\mu}_{1} \tilde{O}_{1} \tilde{\mu}_{2}\right)=\tilde{\mu}_{1} \tilde{O}_{1} \tilde{\mu}_{2}$. Berdasarkan Definisi 2.9(4) dan Definisi 3.7(1),

$$
\begin{aligned}
& \left(\tilde{\mu}_{1} \oplus \tilde{\mu}_{2}\right) \cap\left(\tilde{\mu}_{1} \tilde{O}_{1} \tilde{\mu}_{2}\right) \\
& =\left(\bigcup_{\tilde{\gamma}_{1} \in \tilde{\mu}_{1}, \tilde{\gamma}_{2} \in \tilde{\mu}_{2}}\left[\tilde{\gamma}_{1}^{L}+\tilde{\gamma}_{2}^{L}-\tilde{\gamma}_{1}^{L} \cdot \tilde{\gamma}_{2}^{L}, \tilde{\gamma}_{1}^{U}+\tilde{\gamma}_{2}^{U}-\tilde{\gamma}_{1}^{U} \cdot \tilde{\gamma}_{2}^{U}\right]\right) \\
& \bigcap\left(\bigcup_{\tilde{\gamma}_{1} \in \tilde{\mu}_{1}, \tilde{\gamma}_{2} \in \tilde{\mu}_{2}}\left[\frac{\left|\tilde{\gamma}_{1}^{L}-\tilde{\gamma}_{2}^{L}\right|}{1+\left|\tilde{\gamma}_{1}^{L}-\tilde{\gamma}_{2}^{L}\right|}, \frac{\left|\tilde{\gamma}_{1}^{U}-\tilde{\gamma}_{2}^{U}\right|}{1+\left.\tilde{\gamma}_{1}\right|^{U}-\tilde{\gamma}_{2}^{U} \mid}\right]\right) \\
& =\bigcup_{\tilde{\gamma}_{1} \in \tilde{\mu}_{1}, \tilde{\gamma}_{2} \in \tilde{\mu}_{2}}\left[\min \left\{\tilde{\gamma}_{1}^{L}+\tilde{\gamma}_{2}^{L}-\tilde{\gamma}_{1}^{L} \cdot \tilde{\gamma}_{2}^{L}, \frac{\left|\tilde{\gamma}_{1}^{L}-\tilde{\gamma}_{2}^{L}\right|}{1+\left|\tilde{\gamma}_{1}^{L}-\tilde{\gamma}_{2}^{L}\right|}\right\}\right. \\
& \left.\min \left\{\tilde{\gamma}_{1}^{U}+\tilde{\gamma}_{2}^{U}-\tilde{\gamma}_{1}^{U} \cdot \tilde{\gamma}_{2}^{U}, \frac{\left|\tilde{\gamma}_{1}^{U}-\tilde{\gamma}_{2}^{U}\right|}{1+\left|\tilde{\gamma}_{1}^{U}-\tilde{\gamma}_{2}^{U}\right|}\right\}\right] \\
& =\bigcup_{\tilde{\gamma}_{1} \in \tilde{\mu}_{1}, \tilde{\gamma}_{2} \in \tilde{\mu}_{2}}\left[\frac{\left|\tilde{\gamma}_{1}^{L}-\tilde{\gamma}_{2}^{L}\right|}{1+\left|\tilde{\gamma}_{1}^{L}-\tilde{\gamma}_{2}^{L}\right|}, \frac{\left|\tilde{\gamma}_{1}^{U}-\tilde{\gamma}_{2}^{U}\right|}{1+\left|\tilde{\gamma}_{1}^{U}-\tilde{\gamma}_{2}^{U}\right|}\right] \\
& =\tilde{\mu}_{1} \tilde{O}_{1} \tilde{\mu}_{2} \text {. }
\end{aligned}
$$

Proposisi 3.9. [2] Misalkan $\tilde{\mu}_{1}$ dan $\tilde{\mu}_{2}$ merupakan anggota himpunan lembut kabur hesitant bernilai interval, maka berikut ini berlaku: 
(i) $\left(\tilde{\mu}_{1} \oplus \tilde{\mu}_{2}\right) \cap\left(\tilde{\mu}_{1} \tilde{O}_{2} \tilde{\mu}_{2}\right)=\tilde{\mu}_{1} \tilde{O}_{2} \tilde{\mu}_{2}$,

(ii) $\left(\tilde{\mu}_{1} \oplus \tilde{\mu}_{2}\right) \cup\left(\tilde{\mu}_{1} \tilde{O}_{2} \tilde{\mu}_{2}\right)=\tilde{\mu}_{1} \oplus \tilde{\mu}_{2}$,

(iii) $\left(\tilde{\mu}_{1} \otimes \tilde{\mu}_{2}\right) \cap\left(\tilde{\mu}_{1} \tilde{O}_{2} \tilde{\mu}_{2}\right)=\tilde{\mu}_{1} \tilde{O}_{2} \tilde{\mu}_{2}$,

(iv) $\left(\tilde{\mu}_{1} \otimes \tilde{\mu}_{2}\right) \cup\left(\tilde{\mu}_{1} \tilde{O}_{2} \tilde{\mu}_{2}\right)=\tilde{\mu}_{1} \otimes \tilde{\mu}_{2}$.

Proposisi 3.10. [2] Misalkan $\tilde{\mu}_{1}$ dan $\tilde{\mu}_{2}$ merupakan anggota himpunan lembut kabur hesitant bernilai interval, maka berikut ini berlaku:

(i) $\left(\tilde{\mu}_{1} \oplus \tilde{\mu}_{2}\right) \cap\left(\tilde{\mu}_{1} \tilde{O}_{3} \tilde{\mu}_{2}\right)=\tilde{\mu}_{1} \tilde{O}_{3} \tilde{\mu}_{2}$,

(ii) $\left(\tilde{\mu}_{1} \oplus \tilde{\mu}_{2}\right) \cup\left(\tilde{\mu}_{1} \tilde{O}_{3} \tilde{\mu}_{2}\right)=\tilde{\mu}_{1} \oplus \tilde{\mu}_{2}$,

(iii) $\left(\tilde{\mu}_{1} \otimes \tilde{\mu}_{2}\right) \cap\left(\tilde{\mu}_{1} \tilde{O}_{3} \tilde{\mu}_{2}\right)=\tilde{\mu}_{1} \tilde{O}_{3} \tilde{\mu}_{2}$,

(iv) $\left(\tilde{\mu}_{1} \otimes \tilde{\mu}_{2}\right) \cup\left(\tilde{\mu}_{1} \tilde{O}_{3} \tilde{\mu}_{2}\right)=\tilde{\mu}_{1} \otimes \tilde{\mu}_{2}$.

Proposisi 3.11. [2] Misalkan $\tilde{\mu}_{1}$ dan $\tilde{\mu}_{2}$ merupakan anggota lembut kabur hesitant bernilai interval, maka berikut ini berlaku:

(i) $\left(\tilde{\mu}_{1} \oplus \tilde{\mu}_{2}\right) \cap\left(\tilde{\mu}_{1} \tilde{O}_{4} \tilde{\mu}_{2}\right)=\tilde{\mu}_{1} \tilde{O}_{4} \tilde{\mu}_{2}$,

(ii) $\left(\tilde{\mu}_{1} \oplus \tilde{\mu}_{2}\right) \cup\left(\tilde{\mu}_{1} \tilde{O}_{4} \tilde{\mu}_{2}\right)=\tilde{\mu}_{1} \oplus \tilde{\mu}_{2}$,

(iii) $\left(\tilde{\mu}_{1} \otimes \tilde{\mu}_{2}\right) \cap\left(\tilde{\mu}_{1} \tilde{O}_{1} \tilde{\mu}_{2}\right)=\tilde{\mu}_{1} \tilde{O}_{4} \tilde{\mu}_{2}$,

(iv) $\left(\tilde{\mu}_{1} \otimes \tilde{\mu}_{2}\right) \cup\left(\tilde{\mu}_{1} \tilde{O}_{4} \tilde{\mu}_{2}\right)=\tilde{\mu}_{1} \otimes \tilde{\mu}_{2}$.

\section{Kesimpulan}

Dari pembahasan dapat disimpulkan bahwa operasi irisan dan gabungan pada himpunan lembut kabur hesitant bernilai interval (IVHFSS) memenuhi sifat-sifat aljabar seperti sifat komutatif, sifat assosiatif, sifat distributif dan hukum de Morgan. Selain itu, berdasarkan pembahasan dapat disimpulkan juga terdapat empat operator baru yaitu $\tilde{O}_{1}, \tilde{O}_{2}, \tilde{O}_{3}$ dan $\tilde{O}_{4}$ yang merupakan anggota pada himpunan lembut kabur hesitant bernilai interval (IVHFSS). Untuk dua anggota himpunan lembut kabur hesitant bernilai interval dari himpunan parameter yang sama, penulis dapat menunjukkan sifat-sifat dari keempat operator tersebut.

\section{Ucapan Terima kasih}

Penulis mengucapkan terima kasih kepada Bapak Dr. Admi Nazra, Ibu Dr. Lyra Yulianti, Ibu Dr. Yanita, Bapak Dr. Jenizon, dan Ibu Radhiatul Husna, M.Si yang telah memberikan masukan dan saran sehingga makalah ini dapat diselesaikan dengan baik.

\section{Daftar Pustaka}

[1] Babitha, K.V. dan S.J. Johan 2013. Hesitant fuzzy soft sets. Journal of New Results in Science. 3: $98-107$

[2] Borah, M.J. dan B.Hazarika. 2018. Some operators on interval-valued hesitant fuzzy soft sets. Afr.Mat. https://doi.org/10.1007/s13370-018-0557-5

[3] Chen,N., Xu,Z. dan Xia,M. 2013. Interval valued hesitant preference relations and their applications to group decision making. Knowl. Based Systems. 37: $528-540$ 
[4] Maji, P.K, Biswas, R dan Roy, A.R. 2001. Fuzzy Soft sets. Journal of Fuzzy Mathematics. 9(3): $589-602$

[5] Molodtsov,D.A. 1999. Soft set theory first-result. Comput. Math. Appl. 37: 19 $-31$

[6] Thakur,G.S., Thakur,R. dan Singh,R. 2014. New hesitant fuzzy operators. Fuzzy Inf. Eng.. 6: $379-392$

[7] Torra,V. 2010. Hesitant fuzzy sets. Int.J.Intell.Syst. 25(6): 529 - 539

[8] Wang,J., Li,X. dan Chen,X. 2015.Hesitant fuzzy soft sets with application in multicriteria group decision making problems. Sci. World J. $1-14$

[9] Xu,Z. dan Da,Q.L. 2002. The uncertain OWA operator. Int. J. Intell. Syst. 17: $569-575$

[10] Xu,Z. dan Xia,M. 2011. Distance and similarity measures for hesitant fuzzy sets. Inf. Sci. 181: $2128-2138$

[11] Zadeh,L.A. Fuzzy sets. 1965. Inf. Control. 8: 338 - 353

[12] Zhang,H., Xiaong,L. dan Ma,W. 2015. On interval valued hesitant fuzzy soft sets. Math. Probl. Eng. 17 